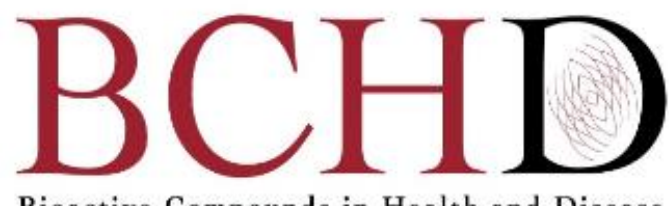

Bioactive Compounds in Health and Disease

\title{
ChoKs as a promising therapeutic target for a variety of pathologies
}

\author{
Tahl Zimmerman, Salam A. Ibrahim*
}

North Carolina Agricultural and Technical State University, Greensboro, NC, United States

*Corresponding Author: Salam A Ibrahim, PhD, Professor, Food and Nutritional Sciences Program, North Carolina Agricultural and Technical State University, Greensboro, NC, United States

Submission Date: November $2^{\text {nd }}, 2021$; Acceptance Date: November 6 ${ }^{\text {th }}, 2021$; Publication Date: November $9^{\text {th }}, 2021$

Please cite this article as: Zimmerman T., Ibrahim S.A. ChoKs as a promising therapeutic target for a variety of pathologies. Bioactive Compounds in Health and Disease 2021; 4(11): 256-259. DOI: https://www.doi.org/10.31989/bchd.v4i11.859

\section{EDITORIAL}

Alterations in cell metabolism represent a common cause of human diseases [1]. Changes in lipid profiles are not an exception to this rule, including those lipids that are produced via the choline kinase (ChoK) pathway. ChoK catalyzes the conversion of choline to phosphocholine via the transfer of a phosphate group from ATP to choline [2]. The formation of phosphocholine is the first step in the Kennedy pathway, which is responsible for the generation of phosphatidylcholine, a critical cell membrane component. ChoK functions as a mediator of cell growth and division and is upregulated in many types of cancers including breast cancer, pancreatic ductal adenocarcinoma, and others [3]. A number of inhibitors have been developed that can block the activity of human ChoK (hChoK) and other eukaryotic
ChoKs, such as those found in parasites. Therefore, ChoK inhibitors (ChoKIs) are promising therapeutics, not only for cancer [4], but for malaria and other diseases caused by parasites [5]. This family of inhibitors are also promising therapeutics for autoimmune diseases such as rheumatoid arthritis (Figure 1) and inflammatory conditions [3].

For example, in the case of rheumatoid arthritis (RA) the ChoKI MN58b was found to block the cell migration and proliferation of fibroblast-like synoviocytes (FLS) in mouse arthritis models. FLS are responsible for cartilage destruction in RA and reverse the effects of FLS abrogated joint inflammation in these models [3]. In addition, ChoKls have shown therapeutic promise in animal models for the treatment of diseases stemming from altered inflammatory responses, such as LPSinduced shock and Muckle-Wells syndrome [3]. 


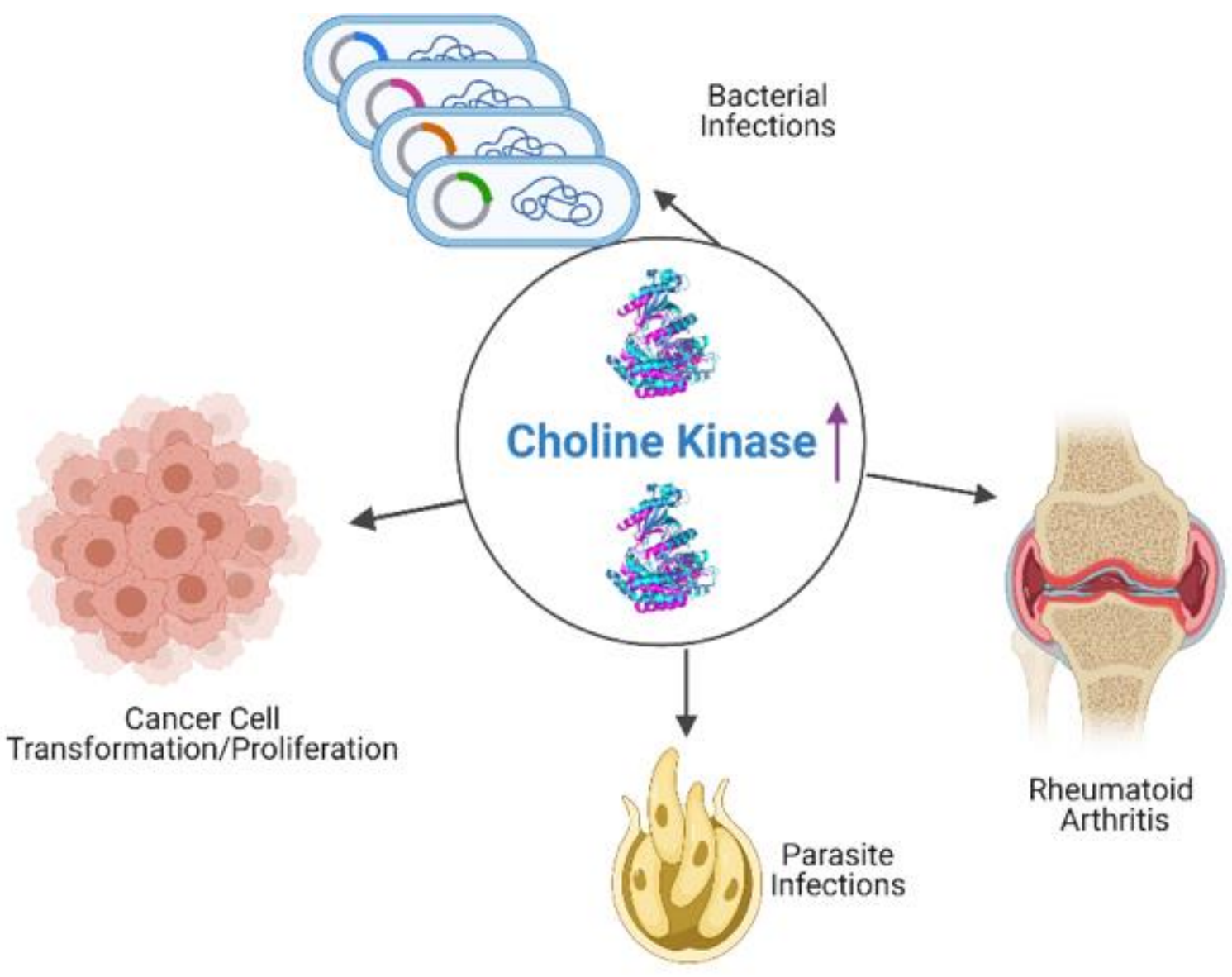

Figure 1: Choline kinase plays a role in a variety of pathologies. Consequently, inhibiting choline kinase is a therapeutic strategy for a wide range of pathologies from bacterial infections to inflammatory disorders.

Importantly one ChoKIs RSM-932A, has been developed to the point of reaching stage I clinical trials for patients with advanced tumours. This suggests that research into ChoKI therapy is beginning to show promise (https://clinicaltrials.gov/ct2/show/NCT01215864) [3].

Despite widespread optimism regarding the benefits of ChoK-based therapies, the therapeutic potential of this system has not yet been fully explored. However, the use of ChoKIs as a possible therapeutic to treat bacterial infections is currently being examined.

\section{Choline kinase inhibition and bacterial pathogens: The} emergence of antibiotic resistant strains of bacterial pathogens is a continuous challenge, which underlines the need for continual research into alternative therapies and the discovery of novel drug targets. One promising strategy is to explore bacterial analogs of eukaryotic drug targets, such as the choline kinase pathway (Figure 1) $[3,6]$. In this way, the same therapies that were developed at considerable expense for eukaryotic systems can be repurposed as antibiotics and antimicrobials for Gram positive bacterial pathogens such as S. pneumoniae.

Choline is an essential nutrient for S. pneumoniae, and choline kinase of $S$. pneumoniae (sChoK) is an essential enzyme. As in eukaryotes, sChoK phosphorylates choline into phosphocholine (Figure 2). However, the subsequent metabolic pathway diverges from the Kennedy pathway as PCho is utilized in the pathways responsible for the synthesis of bacterial teichoic acids such as lipoteichoic acid (LTA) and cell wall teichoic acid (CTA) $[3,6]$. 


\section{Reaction}

Cho + ATP $\rightarrow$ ADP +PCho

$\mathrm{PCho}+\mathrm{ATP} \rightarrow$ diphosphate + CDP-Cho

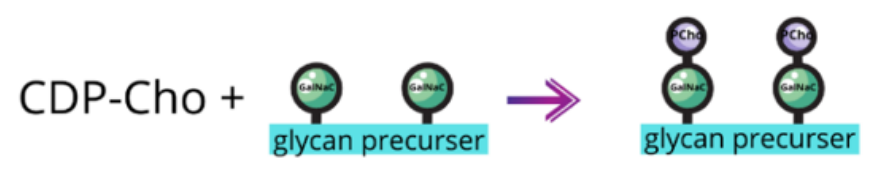

\author{
Enzyme \\ choline kinase \\ phosphophocholine \\ cytidylyl transferase
}

\author{
choline \\ phosphotransferase
}

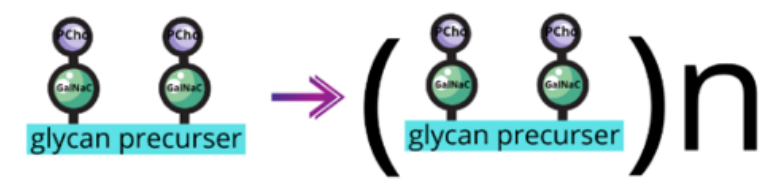

$?$

Figure 2. Choline is an essential nutrient for S. pneumonia and choline kinase of S. pneumoniae (sChoK) is an essential enzyme. As in eukaryotes, the choline kinase of S. pneumoniae (sChoK) phosphorylates choline (Cho) into phosphocholine (PCho). However, the subsequent metabolic pathways diverge from the Kennedy pathway: PCho is utilized in the pathways responsible for the synthesis of bacterial teichoic acids. These are lipoteichoic acid (LTA) and cell wall teichoic acid (CTA) Cytidylyl transferase catalyzes the production of CDP-choline from PCho. Choline phosphotransferases transfer the PCho to the N-acetyl galactosamine (GalNac) residues found on preteichoic acid glycan precursors [7]. The precursors are then assembled by an uncharacterized enzyme into pre-teichoic acid [8]

LTA and CTA are chemically similar, although LTA is embedded in the cell membrane via a glycolipid lipid anchor, and CTA is covalently attached to peptidoglycan molecules in the cell wall via peptidoglycan molecules. Meanwhile, LTA is a known virulence factor, and the LTA synthesis pathway has been shown to be a source of drug targets 8 .

Pcho decoration of the cell surface is required for normal cell-functioning because the Cho moiety anchors choline binding proteins (CBPs) such as murein hydrolases LytA and LytB. LytA and LytB are critical factors for cell division, CBPA which is a determinant of virulence and many others which are involved in colonization and even sepsis. Many other CBPs are involved in colonization and even sepsis. Therefore, the choline metabolic pathways, of which sChoK is an integral part, play an important role in both S. pneumoniae growth and invasion.
With this rationale in mind, the well- known choline analog and competitive ChoKI, $\mathrm{HC}-3$ was studied and found to inhibit sChoK and cell growth with a high $\mathrm{IC}_{50}$ (in the $\mathrm{mm}$ range). This was also found to disrupt lipoteichoic acid production, which was consistent with the cell wall deformations that were observed by scanning electron microscopy. This established sChoK as a putative drug target [9-11]. Later, hChoKIMN58b and RSM-932A were assayed and found to inhibit both sChoK activity and S. pneumoniae cell growth at moderate and low uM concentrations, to modulate lipoteichoic acid production and assembly, and to affect the cell wall [12]. Interestingly, in the case of in vitro studies with purified sChoK, MN58 functions as a competitive inhibitor against both choline and ATP, while RSM932A functions as a competitive and non-competitive inhibitor against choline and ATP, respectively. This result against RSM932A is in sharp contrast to the observed 
uncompetitive behavior of this drug in the case of parasite ChoKs. This would indicate that there are likely enough differences in the largely conserved active sites of the different ChoKs to modulate ChoKI behavior.

This promising outcome suggests that it will likely be possible to rationally design ChoKI that are effective but selective between the different isoforms. In addition, plant derived sChoKIs have been discovered, which leaves open the prospect that dietary compounds could be used as an adjunct to traditional therapeutics (unpublished data). However, much research remains to

\section{REFERENCES}

1. DeBerardinis RJ, Thompson CB. Cellular metabolism and disease: what do metabolic outliers teach us? Cell. 2012 Mar 16;148(6):1132-44. https://doi.org/10.1016/j.cell.2012.02.032

2. Wittenberg j, Kornberg A. Choline phosphokinase. J Biol Chem. 1953 May;202(1):431-44

3. Lacal JC, Zimmerman T, Campos JM. Choline Kinase: An Unexpected Journey for a Precision Medicine Strategy in Human Diseases. Pharmaceutics. 2021 May 25;13(6):788. https://doi.org/10.3390/pharmaceutics13060788

4. Sanchez-Lopez E, Zimmerman T, Gomez del Pulgar T, Moyer MP, Lacal Sanjuan JC, Cebrian A. Choline kinase inhibition induces exacerbated endoplasmic reticulum stress and triggers apoptosis via CHOP in cancer cells. Cell Death Dis. 2013 Nov 28;4(11):e933. https://doi.org/10.1038/cddis.2013.453

5. Zimmerman, T., Moneriz, C., Diez, A., Bautista, J. M., Gómez Del Pulgar, T., Cebrián, A., \& Lacal, J. C. (2013). Antiplasmodial activity and mechanism of action of RSM-932A, a promising synergistic inhibitor of Plasmodium falciparum choline kinase. Antimicrobial agents and chemotherapy, 57(12), 5878-5888.

https://doi.org/10.1128/AAC.00920-13

6. Zimmerman, T., Lacal, J. C., \& Ibrahim, S. A. (2019). Choline Kinase Emerges as a Promising Drug Target in Gram-Positive Bacteria. Frontiers in microbiology, 6, 2146. https://doi.org/10.3389/fmicb.2019.02146 fully understand the physiological effects of these drugs and to fully establish this system as a viable drug target. Ultimately, choline kinase presents us with an important new therapeutic avenue to follow in the development of novel antibiotics, antimicrobials and dietary compounds that could be used to treat or prevent bacterial infections stemming from Gram positive bacteria. Research in this area thus represents a promising new chapter in enhancing our understanding of the role that choline kinase plays in human health.

7. Denapaite D, Brückner R, Hakenbeck R, Vollmer W. Biosynthesis of teichoic acids in Streptococcus pneumoniae and closely related species: lessons from genomes. Microb Drug Resist. 2012 Jun;18(3):344-58. https://doi.org/10.1089/mdr.2012.0026

8. Ginsburg I. Role of lipoteichoic acid in infection and inflammation. Lancet Infect Dis. 2002 Mar;2(3):171-9. https://doi.org/10.1016/s1473-3099(02)00226-8

9. Ibrahim, S. A.; Lacal, J. C.; Zimmerman, T., A dual choline/phosphocholine colorimetric method for measuring the relative strength of inhibitors of choline kinases of Gram-positive pathogens. Food Science and Applied Biotechnology 2018, 1 (2). https://doi.org/10.3390/antibiotics7010024

10. Zimmerman T, Ibrahim S. Choline Kinase, A Novel Drug Target for the Inhibition of Streptococcus pneumoniae. Antibiotics (Basel). 2017 Sep 25;6(4):20. https://doi.org/10.3390/antibiotics6040020

11. Zimmerman T, Ibrahim SA. Parallel Colorimetric Quantification of Choline and Phosphocholine as a Method for Studying Choline Kinase Activity in Complex Mixtures. Antibiotics (Basel). 2018 Mar 17;7(1):24 doi: 10.3390/antibiotics7010024.

12. Zimmerman, T., Chasten, V., Lacal, J.C. et al. Identification and validation of novel and more effective choline kinase inhibitors against Streptococcus pneumoniae. Sci Rep 10, 15418 (2020). https://doi.org/10.1038/s41598-020-72165-6 